\title{
ON ALMOST MAXIMAL RIGHT IDEALS
}

\author{
KWANGIL KOH
}

Abstract. A concept of "a prime ideal" in a commutative ring is extended to a general ring such that it properly includes the class of maximal one sided ideals. Such a right (or left) ideal is called almost maximal. The main theorems in the present paper are as follows:

(1) If $R$ is a ring with 1 then a right ideal $I$ is almost maximal if and only if $\operatorname{Hom}_{R}\left([R / I]_{0},[R / I]_{0}\right)$ is a division ring where $[R / I]_{0}$ is the quasi-injective hull of $R / I$, and for any nonzero submodule $N$ of $R / I$ there is a nonzero endomorphism $f$ of $R / I$ such that $f(R / I) \subset N$.

(2) If $R$ is a ring with 1 then $R$ is a right noetherian ring and every almost maximal right ideal is maximal if and only if $R$ is a right artinian ring.

1. If $R$ is a ring and $I$ is a right ideal of $R$, let $N(I)=\{x \in R \mid x I \subset I\}$ and $N^{*}(I)=\{x \in N(I) \mid x y \in I$ if and only if $y \in I\} . N(I)$ is called the "normalizer of $I$ " in $R$ and it is the largest subring of $R$ in which $I$ is contained as an ideal. (Refer $[4$, p. 25].) Let $R \backslash I$ denote the (set) complement of $I$ in $R$. We define a right ideal $I$ which is not $R$ to be almost maximal if and only if

(A1) for any $a, b \in R \backslash I$ there are $r_{1}, r_{2} \in R$ and $c \in N^{*}(I)$ such that $a r_{1} \equiv b r_{2} \equiv c \bmod I$,

(A2) if $a \in R \backslash I$, then either $a \in N(I)$ or $a r \equiv a i \bmod I$ for some $r \in R \backslash I$ and $i \in I$.

If $R$ is a commutative ring then (A2) is true always for any ideal $I$ and the condition (A1) holds true if and only if $I$ is a prime ideal.

The purpose of this paper is to prove the following theorems:

(1) If $R$ is a ring with 1 then a right ideal $I$ is almost maximal if and only if

(i) $\operatorname{Hom}_{R}\left([R / I]_{0},[R / I]_{0}\right)$ is a division ring, where $[R / I]_{0}$ is the quasi-injective hull of $R / I$,

(ii) if $N$ is a nonzero submodule of $R / I$ then there is a nonzero endomorphism $f$ in $\operatorname{Hom}_{R}(R / I, R / I)$ such that $f(R / I) \subset N$.

(2) If $R$ is a ring with 1 then $R$ is a right noetherian ring and every almost maximal right ideal is maximal if and only if $R$ is a right artinian ring.

(2) is a generalization of Theorem 2 of $[7$, p. 205].

Received by the editors July 25, 1969.

AMS Subject Classifications. Primary 1620, 1625, 1640.

Key Words and Phrases. Normalizer, quasi-injective hull, noetherian ring, artinian ring, Goldie ring, strongly regular ring. 
2. Lemma 2.0. A proper right ideal $I$ in a ring $R$ is almost maximal right ideal if and only if the right $R$-module $M=R / I$ has the following properties:

(i) Every nonzero submodule of $M$ is large.

(ii) $m \neq 0$ in $M$ there exists $r \in R$ such that $\operatorname{Ann}(m r)=I$.

(iii) $m$ in $M$, either $\operatorname{Ann}(m) \supset I$ or $\operatorname{Ann}(m) \nsubseteq I$, where for any $m \in M$, $\operatorname{Ann}(m)=\{r \in R \mid m r=0\}$.

Proof. Suppose $I$ is almost maximal. By (A1), (i) and (ii) are true. To see (iii), assume $\operatorname{Ann}(m) \subset I$. Let $m=a+I$ such that $a \notin I$. Let $J=\operatorname{Ann}(m)$. If $x \in I$ such that $a x \notin I$ then $a \notin N(I)$. Hence by (iii) there exists $y \in R \backslash I$ such that $a(y-i) \in I$ for some $i \in I$. Therefore $y-i \in J \subset I$ and $y \in I$. This is impossible. Thus $I \subset \operatorname{Ann}(m)$. Conversely, suppose $R \backslash I$ satisfies (i), (ii) and (iii). Let $a, b \in R \backslash I$. Then by (i) there exist $r_{1}$ and $r_{2}$ in $R$ such that $m r_{1}=m r_{2}$; i.e., $a r_{1}=b r_{2}$ mod $I$. Now by (ii) there exists $r \in R$ such that $\operatorname{Ann}\left(m r_{1} r\right)=I$ $=\operatorname{Ann}\left(m r_{2} r\right)$. Hence $a r_{1} r \equiv a r_{2} r \equiv c \bmod I$ for some $c \in N^{*}(I)$. Now let $a \in R \backslash I$ such that $a \in N(I)$. Then $\operatorname{Ann}(a+I) D I$. Hence by (iii) $\operatorname{Ann}(a+I) \nsubseteq I$. Let $x \in \operatorname{Ann}(a+I)$ such that $x \notin I$ and let $i \in I$ such that $a i \in I$. Then $a x \equiv a i \bmod I$.

CoRollary. If I is a maximal modular right ideal then $I$ is almost maximal.

Proof. The right $R$-module $R / I$ is simple, and it trivially satisfies the conditions (i), (ii) and (iii) of Lemma 2.0. Thus $I$ is almost maximal.

Lemma 2.1. Let $R$ be a ring and $I$ be a right ideal such that $I \neq R$. Then $I$ is an almost maximal right ideal if and only if

(i) $I$ is irreducible in the sense that if $A$ and $B$ are right ideals such that $A \supset I, B \supset I, A \neq I$ and $B \neq I$ then $A \cap B \neq I$.

(ii) if $a^{-1} I$ contains $I$ properly for some $a \in R$ then $a \in I$,

(iii) if $J$ is a right ideal of $R$ which properly contains $I$ and $a \in R$, then, IC $a^{-1} J$ implies $a^{-1} J$ properly contains $I$,

(iv) if $J$ is a right ideal which contains $I$ properly then $N(I) \cap J \neq I$.

Proof. Suppose $I$ is almost maximal. We observe that (i) and (iv) are direct consequence of (A1). To see (ii), consider the right $R$ module $R / I$. If $a \in R$, let $[a]=a+I$ and $\operatorname{Ann}([a])=\{r \in R \mid$ ar $\in I\}$. Suppose Ann ([a]) $I$ and $\operatorname{Ann}([a]) \neq I$. We claim that $a \in I$. If $a \notin I$ then, by (ii) of Lemma 2.0, there is $r^{\prime}$ in $R$ such that $\operatorname{Ann}\left(\left[a r^{\prime}\right]\right)=I$. Hence Ann $([a]) \neq R$. Let $b \in R$ such that $b \in \operatorname{Ann}([a])$. Then, again, by (ii) of Lemma 2.0 there is $r_{0} \in R$ such that $\operatorname{Ann}\left(\left[a b r_{0}\right]\right)=I$. By 
(iii) of Lemma 2.0, either $\operatorname{Ann}\left(\left[b r_{0}\right]\right) \nsubseteq I$ or $\operatorname{Ann}\left(\left[b r_{0}\right]\right) \supset I$. Since $\operatorname{Ann}\left(\left[b r_{0}\right]\right) \subset \operatorname{Ann}\left(\left[a b r_{0}\right]\right)=I$, this means that $\operatorname{Ann}\left(\left[b r_{0}\right]\right) \supset I$ and therefore $\operatorname{Ann}\left(\left[b r_{0}\right]\right)=I$. By (i) of Lemma 2.0, $b r_{0} R+I / I$ $\cap \operatorname{Ann}([a]) / I \neq 0$. Let $\left[b r_{0} r^{\prime}\right] \equiv \bar{d} \not \equiv 0$ for some $r^{\prime} \in R$ and $d \in \operatorname{Ann}([a])$. Since $\operatorname{Ann}\left(\left[b r_{0}\right]\right)=I, \quad r^{\prime} \notin I$. However $b r_{0} r^{\prime}-d \in I$ implies that $a b r_{0} r^{\prime} \equiv a d \equiv 0$ and $r^{\prime} \in \operatorname{Ann}\left(\left[a b r_{0}\right]\right)=I$. This is a contradiction. Now to see (iii), suppose $a^{-1} J=I$ for some $a \in R$ and some right ideal $J$ which contains $I$ properly. If $a \in I$ then $a^{-1} J=R$. So suppose $a \notin I$. Let $j \in J$ such that $j \notin I$. Then by (A1) there exist $r_{1}, r_{2} \in R$ and $c \in N^{*}(I)$ such that $a r_{1} \equiv j r_{2} \equiv c$. Since $r_{1} \in a^{-1} J$ and $a^{-1} J=I, r_{1} \in I$. Therefore $a \notin N(I)$ for if $a \in N(I)$ then $c \notin N^{*}(I)$. Hence by (A2), there exist $r^{\prime} \in R \backslash I$ and $i \in I$ such that $a r^{\prime} \equiv a i$. This means that $\left(r^{\prime}-i\right) \in a^{-1} J=I$ and $r^{\prime} \in I$. This is impossible. Conversely, assume conditions (i), (ii), (iii), and (iv). Then the conditions (i) and (ii) of Lemma 2.0 are satisfied. To see the condition (iii) of Lemma 2.0 is satisfied, one observes that if $\operatorname{Ann}([a]) D I$ then the right ideal $a I+I$ contains $I$ properly and $a^{-1}(a I+I) \supset I$. Hence by (iii), there exists $x \in a^{-1}(a I+I)$ such that $x \in I$ and $a x=a i+i^{\prime}$ for some $i, i^{\prime} \in I$. Therefore if $\operatorname{Ann}([a]) \subset I$ then $x-i \in \operatorname{Ann}([a]) \subset I$ and $x \in I$. This is impossible. Hence $\operatorname{Ann}([a]) \nsubseteq I$. Thus by Lemma 2.0, $I$ is almost maximal.

Lemma 2.2. Suppose $I$ is a right ideal of $R$ such that $N(I) \neq I$ and $R^{2} \subsetneq I$. If $\operatorname{Hom}_{R}\left([R / I]_{0},[R / I]_{0}\right)$ is a division ring and for any nonzero submodule $N$ of $R / I$ there is a nonzero $f \in \operatorname{Hom}_{R}(R / I, R / I)$ such that $f(R / I) \subset N$ then $N^{*}(I)=N(I) \backslash I$.

Proof. Clearly $N^{*}(I) \subset N(I) \backslash I$. Let $c \in N(I) \backslash I$. Let

$$
J=\{x \in R \mid x R \subset I\} .
$$

Then $J$ is a right ideal of $R$. Define $t_{c}(r+I)=c r+I$ for any $r \in R$. Then $t_{c} \in \operatorname{Hom}_{R}(R / I, R / I)$. If $t_{c}$ is a nonzero homomorphism then the kernel of $t_{c}$ is zero since $\operatorname{Hom}_{R}\left([R / I]_{0},[R / I]_{0}\right)$ is a division ring and hence $c \in N^{*}(I)$. So suppose $t_{c}$ is a zero homomorphism. Then $c \in J$ and $J / I$ is a nonzero submodule of $R / I$. Hence by hypothesis, there exists a nonzero $f \in \operatorname{Hom}_{R}(R / I, R / I)$ such that $f(R / I) \subset J / I$. Let $x \in R \backslash I$ such that $x R \nsubseteq I$. Then $f(x+I) \neq 0$ since the kernel of $f$ is zero and hence $f(x+I)=j+I$ for some $j \in J \backslash I$. Therefore $f(x+I) R=0$ and $x R \subset I$. This is impossible. Thus $t_{c}$ is not a zero homomorphism and $c \in N^{*}(I)$.

THEOREM 2.3. Let $I$ be a right ideal of a ring $R$ such that $N(I) \neq I$ and $R^{2} \subset I$. Then $I$ is an almost maximal right ideal if and only if 
(i) $\operatorname{Hom}_{R}\left([R / I]_{0},[R / I]_{0}\right)$ is a division ring,

(ii) if $N$ is a nonzero submodule of $R / I$ then there is a nonzero $f \in \operatorname{Hom}_{R}(R / I, R / I)$ such that $f(R / I) \subset N$.

Proof. If $I$ is an almost maximal right ideal of $R$ then by Lemma 2.1 and $\left[6\right.$, Theorem 3.2, p. 67], $\operatorname{Hom}_{R}\left([R / I]_{0},[R / I]_{0}\right)$ is a division ring. Let $N$ be a nonzero submodule of $R / I$. Then $N=J / I$ for some right ideal $J$. Let $j \in J, j \in I$. By (A1) there exist $r \in R$ and $c \in N^{*}(I)$ such that $j r \equiv c \bmod I$. Define $t_{c}(x+I)=c x+I$ for any $x \in R$. Then $t_{c} \cap \operatorname{Hom}_{R}(R / I, R / I)$ and $t_{c}(R / I) \subset N$. Conversely, assume (i) and (ii). Let $c \in N(I)$ and $c \notin I$. Then by Lemma 2.2, $c \in N^{*}(I)$. Let $a, b \in R \backslash I$. Let $N_{1}=a R+I / I$ and $N_{2}=b R+I / I$. Then $N_{1}, N_{2}$ are nonzero submodules of $R / I$, since if either $a R \subset I$ or $b R \subset I$ then $a$ or $b$ would be an element of $N(I) \backslash I$ which is not an element of $N^{*}(I)$ which is, of course, absurd since $N^{*}(I)=N(I) \backslash I$ by Lemma 2.2. If $N_{1} \cap N_{2}=0$, we can define an $R$-homomorphism from $N_{1}+N_{2}$ into $R / I$ by $f: n_{1}+n_{2} \rightarrow n_{2}, n_{2} \in N_{2}$ and $n_{2} \in N_{2}$. An extension $\tilde{f}$ of $f$ to $[R / I]_{0}$ would have a nonzero kernel, which is, of course, impossible since $\operatorname{Hom}_{R}\left([R / I]_{0},[R / I]_{0}\right)$ is a division ring. Therefore $N=N_{1} \cap N_{2}$ is a nonzero submodule of $R / I$. Hence there is a nonzero homomorphism $f \in \operatorname{Hom}_{R}(R / I, R / I)$ such that $f(R / I) \subset N$. Let $f(c+I)=c^{\prime}+I$. Then $c^{\prime} \in N(I) \backslash I=N^{*}(I)$ since $c \in N(I) \backslash I$ and the kernel of $f$ is zero. Since $c^{\prime}+I \in N, c^{\prime}+I=a r_{1}+I=b r_{2}+I$ for some $r_{1}, r_{2}$ in $R$. Thus $a r_{1} \equiv b r_{2} \equiv c^{\prime} \bmod I$. This proves (A1). To prove (A2), let $a \in R \backslash I$ and $a \notin N(I)$. Let $J=a I+I$. Then $J$ contains $I$ properly and $a^{-1} J \supset I$. If $I=a^{-1} J$, define $\phi: a r+I \rightarrow r+I$ for all $r \in R$. If $a r_{1}-a r_{2} \in I$ for some $r_{1}, r_{2} \in R$ then $r_{1}-r_{2} \in a^{-1} I \subset a^{-1} J=I$. Hence $r_{1}-r_{2} \in I$ and $\phi$ is a nonzero homomorphism of the submodule $(a+I) R$ onto $R / I$. Therefore, an extension $\tilde{\phi}$ of $\phi$ to $[R / I]_{0}$ is an isomorphism since $\operatorname{Hom}_{R}\left([R / I]_{0},[R / I]_{0}\right)$ is a division ring. Hence the kernel of $\phi$ is zero and $a I \subset I$. Hence $\phi^{-1}(J / I) \cap J / I \neq 0$ and $a j \in J$ for some $j \notin I$. This contradicts the assumption that $I=a^{-1} J$. Thus $a^{-1} J$ must contain $I$ properly and $a r^{\prime} \equiv a i \bmod I$ for some $r^{\prime} \notin I$ and some $i \in I$.

REMARK 2.4. In case $R$ is a semiprime ring with zero singular (right) ideal and $U$ is a uniform right ideal, that is, every pair of nonzero right ideals of $R$ which are contained in $U$ has a nonzero intersection then for any $0 \neq x \in U$ the right annihilators of $x$ is an almost maximal right ideal. This can be seen as follows. First we note that $x R \cong R /(x)^{r}$ where $(x)^{r}$ is the right annihilators of $x$. Hence by $\left[5,1.7\right.$, p. 263], $\operatorname{Hom}_{R}\left([R /(x)]_{0}^{r},[R /(x)]_{0}^{r}\right)$ is a division ring. If $N$ is a nonzero submodule of $R /(x)^{r}$, then $N=J /(x)^{r}$ for some right ideal $J$. Since $R$ is semiprime and $x J \neq 0, J x \neq 0$. Let $j x \neq 0 \in J$ for 
some $j$. Then $(j x)^{r}=(x)^{r}$ since the right singular ideal of $R$ is zero. Hence the map: $t_{j x}\left(r+(x)^{r}\right)=j x r+(x)^{r}$ for all $r \in R$ is a nonzero homomorphism of $R /(x)^{r}$ into $R /(x)^{r}$ and $t_{j x}\left(R /(x)^{r}\right) \subset J /(x)^{r}$.

3. Lemma 3.1. Let $R$ be a semiprime right Goldie ring. Then every almost maximal right ideal of $R$ is maximal if and only if $R$ is a semisimple artinian ring.

Proof. If $R$ is a semisimple artinian ring then every module is injective. Hence if $I$ is an almost maximal right ideal of $R$ such that $R / I$ contains a nonzero submodule then it must be a direct summand of $R / I$. Since every pair of nonzero submodules of $R / I$ has a nonzero intersection by (i) of Lemma $2.1, R / I$ has no direct summand except zero and itself. Thus $R / I$ is simple and $I$ is a maximal right ideal of $R$. Conversely, assume that every almost maximal right ideal of $R$ is maximal. Let $U$ be a uniform right ideal of $R$. By Remark 2.4, if $0 \neq x \in U$ then $(x)^{r}$ is an almost maximal right ideal of $R$, hence it is maximal. Therefore $x R$ is a minimal right ideal of $R$. This is true for any $0 \neq x \in U$ and $U$ is uniform, $U$ must be a minimal right ideal. Now let $U_{1}, U_{2}, \cdots, U_{n}$ be $n$ uniform right ideals in $R$ such that $\sum_{i=1}^{n} \oplus U_{i}$ is a large right ideal of $R$. Then there is an element $a$ in $\sum_{i=1}^{n} \oplus U_{i}$ such that $(a)^{r}=\{0\}$ by [3, Theorem 3.9]. Let $S$ be the right socle of $R$. Then $\sum_{i=1}^{n}+U_{i} \subset S$. Let $Q(R)$ be the complete right quotient ring of $R$ so that $a^{-1} \in Q(R)$. Let $K=\left\{r \in R \mid a^{-1} r \in R\right\}$. Then $K$ is a large right ideal of $R$ and $S \subset K$. Since $a^{-1} S \subset R, a^{-1} U \subset S$ for any minimal right ideal $U$. Therefore $a^{-1} S \subset S$ and $1 \in S$. Thus $S=R$ and $R$ is semisimple artinian.

Lemma 3.2. If $I$ is an almost maximal right ideal of a ring $R$ then $p(I)=\{x \in R \mid R x \subset I\}$ is a prime ideal.

Proof. Let $A$ and $B$ be ideals of $R$ such that $A B \subset p(I)$. If $A \nsubseteq p(I)$ and $B \nsubseteq D(I)$ then $A \nsubseteq I$ and $B \nsubseteq I$ since $p(I)$ is the largest ideal of $R$ which is contained in $I$. Let $a \in A \backslash I$ and $b \in B \backslash I$. Then by (A1) there exist $r_{1}, r_{2}$ and $c \in N^{*}(I)$ such that $a r_{1} \equiv b r_{2} \equiv c \bmod I$. This means that $a r_{1} \cdot b r_{2} \equiv c^{2} \bmod I$ since $N(I) / I$ is a ring and $c^{2} \in I$. This is impossible since $c^{2} \in N^{*}(I)$ and $N^{*}(I) \cap I=\varnothing$.

Lemma 3.3. Let $I$ be a right ideal of a ring $R$ and let $S$ be an ideal of $R$ such that $I \supset S$. Then $I / S$ is an almost maximal right ideal of $R / S$ if and only if $I$ is an almost maximal right ideal of $R$.

Proof. Straightforward. 
TheOREM 3.4. Let $R$ be a ring with 1 . Then the following two statements are equivalent:

(i) $R$ is right noetherian and every almost maximal right ideal of $R$ is maximal.

(ii) $R$ is right artinian.

Proof. Assume (ii). Then $R$ is right noetherian (refer [1, Theorem 15 , p. 48]). Let $r(R)$ be the prime radical of $R$, that is, the intersection of prime ideals in $R$. If $I$ is an almost maximal right ideal of $R$ then $I \supset r(R)$ by Lemma 3.2. By Lemma 3.3, $I / r(R)$ is an almost maximal right ideal of $R / r(R)$. Since $R / r(R)$ is semisimple artinian, by Lemma 3.1, $I / r(R)$ is a maximal right ideal of $R / r(R)$. Hence $I$ is a maximal right ideal of $R$. Conversely, assume (i). Since any almost maximal right ideal of $R$ is maximal and $R / r(R)$ is right noetherian, by Lemma 3.3 and Lemma 3.1, $R / r(R)$ is semisimple artinian. Therefore $J(R)$, the Jacobson radical of $R$, is equal to $r(R)$ and $J(R)$ is an nil ideal. Since $R$ is right noetherian and $J(R)$ is nil, $J(R)$ is nilpotent. Therefore by [2, Theorem (S. U. Chase), p. 189], $R$ is right artinian.

PROPOSITION 3.5. If $R$ is a strongly regular ring (i.e. if $a \in R$ then there is $x \in R$ such that $\left.a^{2} x=a x a=a\right)$ then an almost maximal right ideal of $R$ is maximal.

Proof. If $R$ is a strongly regular ring then for any $a \in R, a R$ is generated by a central idempotent element. Hence any right ideal of $R$ is an ideal. Hence if $I$ is an almost maximal right ideal of $R$ then $N(I)=R$ and $R / I$ is isomorphic to a subring of $\operatorname{Hom}_{R}(R / I, R / I)$ which is an integral domain by Theorem 2.3. Now if $a \in R \backslash I$ then there is $x \in R$ such that $a x \in R \backslash I$ and $(a x)(a x)=a x$. Therefore, the map: $t_{a x}(r+I)=a x r+I$ for any $r \in R$ is the identity map since $0 \neq t_{a x} \in \operatorname{Hom}_{R}(R / I, R / I)$ and $t_{a x}^{2}=t_{a x}$. Therefore $R / I$ is a division ring and $I$ is maximal.

Acknowledgment. The author is indebted to the referee for many helpful suggestions. In particular, the statement of Lemma 2.0 is entirely the referee's.

\section{REFERENCES}

1. N. J. Divinsky, Rings and radicals, Mathematical Expositions, no. 14, Univ. of Toronto Press, Toronto, 1965. MR 33 \#5654.

2. C. Faith, Rings with ascending conditions on annihilators, Nagoya Math. J. 27 (1966), 179-191. MR 33 \#1328. 
3. A. W. Goldie, Semi-prime rings with maximum condition, Proc. London Math. Soc. (3) 10 (1960), 201-220. MR 22 \#2627.

4. N. Jacobson, Structure of rings, 2nd rev. ed., Amer. Math. Soc. Colloq. Publ., vol. 37, Amer. Math. Soc., Providence, R. I., 1964. MR 36 \#5158.

5. R. E. Johnson and E. T. Wong, Quasi-injective modules and irreducible rings, J. London Math. Soc. 36 (1961), 260-268. MR 24 \#A1295.

6. K. Koh and A. C. Mewborn, A class of prime rings, Canad. Math. Bull. 9 (1966), 63-72. MR 33 \#5656.

7. Oscar Zariski and P. Samuel, Commutative algebra. Vol. 1, The University Series in Higher Mathematics, Van Nostrand, Princeton, N. J., 1958. MR 19, 833.

North Carolina State University, Raleigh, North Carolina 27607 